Ann. Biol. anim. Bioch. Biophys., 1979, 19 (3 B), 757-761.

\title{
Mesure de l'absorption du cholestérol chez le rat. Identité des résultats obtenus par la méthode d'équilibre isotopique et la méthode de Zilversmit
}

par C. LUTTON

Laborotoire de Physiologie de la Nutrition Université Paris XI, Bâtiment 447 91405 Orsay Cedex.

Summary. Identity of results obtained with isotopic equilibrium and isotopic ratio methods for the measurement of cholesterol absorption in rats.

Cholesterol turnover rates, including that of absorption, may be measured in vivo in very good physiological conditions using the isotopic equilibrium method. As every balance method, it necessitates complete fecal collection and analysis. Zilversmit has devised a simple method for cholesterol adsorption measurement using a single blood or plasma sample following simultaneous isotopic doses of orally and intravenously administered cholesterol. The Zilversmit isotopic ratio method (IRM) has been applied to two groups of eight 3 and 6-month old rats fed a basal semipurified diet. One month after the IRM, the rats of the first group were studied with the isotopic equilibrium method (IEM). At the end of the IEM, the rats were 6 months old, the age of the rats of the second group. No differences were seen between the cholesterol absorption coefficients (measured by IRM) of rats in the first group $(73.4 \pm 2.4$ p. 100) and those in the second group $(72.4 \pm 4.3$ p. 100) or when they were measured by IEM $(69.2 \pm 1.5 \mathrm{p} .100)$. The two methods have been critically compared.

\section{Introduction.}

Il existe peu de méthodes de mesure in vivo de l'absorption du cholestérol satisfaisantes pour le physiologiste. Le plus classique consiste à ajouter du cholestérol radioactif à un repas et à collecter les fèces pendant le temps nécessaire au recouvrement complet des molécules marquées non absorbées (Borgström, 1968). Apparentée à ce type de technique, la méthode d'équilibre isotopique permet une mesure des processus de renouvellement du cholestérol dont l'excrétion fécale et l'absorption, dans des conditions physiologiques excellentes (Lutton et Chevallier, 1972). Elle nécessite aussi de recueillir les fèces. Une méthode simple de mesure de l'absorption du cholestérol ne faisant appel qu'à l'analyse de la radioactivité du cholestérol d'un échantillon de sang ou de plasma a été décrite par Zilversmit en 1972. Ses résultats semblent compa- 
rables à ceux obtenus par la technique de collection des fèces après administration orale de cholestérol marqué chez le Rat (Zilversmit, 1972), le singe (Kritchevsky, Winter et Davidson, 1974 ; Corey et Hayes, 1975), l'homme (Samuel, Crouze et Ahrens, 1978) ou le cobaye (Traber ef Ostwald, 1978). Dans son étude, Zilversmit s'étonne des valeurs parfois élevées obtenues par la méthode d'équilibre isotopique, suggérant ainsi qu'elle surestime le coefficient d'absorption du cholestérol. Afin d'éclaircir ce différend, nous avons donc entrepris de comparer les deux méthodes en utilisant des rats placés dans des conditions semblables.

\section{Matériel ef méthodes.}

1) Animaux et régime. - Seize rats mâles, de souche Wistar, ingèrent depuis leur sevrage un régime semi-synthétique dont la composition est : saccharose (53 p. 100), caséine (23 p. 100), saindoux (9,2 p. 100), huile de noix (0,2 p. 100), lait (4 p. 100), sels minéraux ( 5 p. 100), agar-agar $(2,3$ p. 100$)$, levure de bière $(2,3$ p. 100$)$, vifamines $(0,2$ p. 100), cystine (0,2 p. 100). Sa concentration en cholestérol est de 0,015 p. 100. La technique de Zilversmit est appliquée à un premier lot de 8 rats âgés de 3 mois sur lesquels, un mois après, un équilibre isotopique est réalisé. Celui-ci dure 8 semaines. Les rats sont donc âgés de 6 mois à la fin de l'équilibre isotopique. Aussi, la méth ode de Zilversmit a été également appliquée à 8 autres rats âgés de 6 mois (lot 2).

2) Méthode de Zilversmit. - Après un jeûne de $16 \mathrm{~h}$, une dose orale de $\left(4^{14} \mathrm{C}\right)$ cholestérol (1-2 $\mu \mathrm{Ci}$ ) est donnée par tubage gastrique suivie de l'injection intrajugulaire de $\left({ }^{3} \mathrm{H}\right)$ cholestérol $(2-4 \mu \mathrm{Ci})$ effectuée sous anesthésie à l'éther (Zilversmit et Hughes, 1974). Une prise de sang par ponction au sinus veineux est effectuée 48 et $96 \mathrm{~h}$ après l'opération chez les rats du lot 1, et après $96 \mathrm{~h}$ par ponction aortique chez ceux du lot 2 .

Calcul du coefficient d'absorption : ll est donné par le rapport constant à partir du deuxième jour (Zilversmit et Hughes, 1974) :

Radioactivité $\left({ }^{14} \mathrm{C}\right)$ cholestérol de l'échantillon

(en p. 100 de la dose orale)

Radioactivité $\left({ }^{3} \mathrm{H}\right)$ cholestérol de l'échantillon

100.

(en p. 100 de la dose intraveineuse)

3) Méthode d'équilibre isotopique. - Les rats sont sacrifiés après 8 semaines d'un double équilibre isotopique obtenu par injections sous-cutanées quotidiennes de $\left({ }^{3} \mathrm{H}\right)$ cholestérol $(0,2 \mathrm{mg}, 1 \mu \mathrm{Ci} / \mathrm{jour})$ el par adjonction à l'alimentation de $\left(4^{14} \mathrm{C}\right)$ cholestérol en quantité traceuse ( $25 \mu \mathrm{Ci} / \mathrm{kg}$ de régime) (Lutton et Chevallier, 1972). La radioactivité spécifique du cholestérol mobile (plasma, globules rouges, ...) reste constante pour les deux isotopes après 30 jours de même que la radioactivité éliminée. Les méthodes chimiques et isotopiques ont été décrites (Chevallier et Mathé, 1964).

Calcul du coefficient d'absorption : Dans le cas de l'équilibre isotopique par injections de $\left({ }^{3} \mathrm{H}\right)$ cholestérol, seul le cholestérol d'excrétion et ses produits de transformation sont radioactifs. En conséquence, la masse de chole stérol fécal dont l'origine correspond à une excrétion $\left(\mathrm{m}_{\mathrm{EF}}\right)$ est obtenue par le quotient de la radioactivité $\left({ }^{3} \mathrm{H}\right)$ 
des stérols neutres fécaux $\left(R_{F}^{\prime}\right)$ par la radioactivité spécifique du $\left({ }^{3} \mathrm{H}\right)$ cholestérol plasmatique $\left(\mathbf{r}^{\prime} \mathbf{p}\right)$.

$$
\mathrm{m}_{\mathrm{EF}}=\frac{\mathrm{R}_{\mathrm{F}}^{\prime}}{\mathrm{r}_{\mathbf{P}}^{\prime}}
$$

Dans le cas où l'équilibre isotopique est réalisé par addition de $\left({ }^{14} \mathrm{C}\right)$ cholestérol à l'alimentation, la radioactivité ${ }^{14} \mathrm{C}$ des stérols neutres fécaux $\left(R_{F}\right)$ résulte de la présence du cholestérol alimentaire non absorbé $\left(m_{N A}\right)$ de radioactivité spécifique égale à celle du cholestérol ingéré $\left(r_{\mathrm{I}}\right)$ et du cholestérol excrété $\left(\mathrm{m}_{\mathrm{EF}}\right)$ ayant la radioactivité spécifique du $\left({ }^{14} \mathrm{C}\right)$ cholestérol plasmatique $\left(r_{\mathrm{P}}\right)$. On a :

$$
R_{F}=\left(m_{N A} \times r_{I}\right)+\left(m_{E F} \times r_{P}\right) .
$$

Obtenant $m_{E F}$ à partir de l'équation (1), on en déduit la masse de cholestérol non absorbé $\left(\mathrm{m}_{\mathrm{NA}}\right)$ par l'équation (2). Connaissant, par ailleurs, la masse de cholestérol ingéré $\left(m_{I}\right)$, on en déduit la masse de cholestérol absorbé $\left(m_{A}\right)$ et le coefficient d'absorption $\left(m_{A} / m_{I} \times 100\right)$.

\section{Résultats et discussion.}

Les coefficients d'absorption (CA) du cholestérol mesuré chez les rats 1-8 par la méthode de Zilversmit ou pendant la huitième semaine d'équilibre isotopique sont portés dans le tableau. Aucune différence n'est observée entre les résultats obtenus par ces deux méthodes sur les mêmes rats étudiés à trois mois d'intervalle. De même, le CA des rats 9-16 âgés de 6 mois mesuré par la méthode de Zilversmit (72,4 p. $100 \pm 4,3$ p. 100) ne diffère pas de celui obtenu par la méthode de l'équilibre isotopique sur les rats $1-8$ du même âge $(69,2 \pm 1,5$ p. 100).

\section{TABLEAU}

Coefficient d'absorption du cholestérol

mesuré par les méthodes de Zilversmit et d'équilibre isotopique

\begin{tabular}{ccccc}
\hline Rat & $\begin{array}{c}\text { Méthode de } \\
\text { Zilversmit }\end{array}$ & $\begin{array}{c}\text { Méthode } \\
\text { d'équilibre } \\
\text { isotopique }\end{array}$ & Rat & $\begin{array}{c}\text { Méthode de } \\
\text { Zilversmit }\end{array}$ \\
\hline 1 & $66,5\left(^{a}\right)$ & $76,5\left(^{(0)}\right.$ & 9 & 82,2 \\
2 & 73,6 & 67,7 & 10 & 83,2 \\
3 & 81,8 & 65,1 & 11 & 74,0 \\
4 & 82,7 & 71,5 & 12 & 46,9 \\
5 & 66,5 & 63,1 & 13 & 77,7 \\
6 & 78,2 & 68,6 & 14 & 64,5 \\
7 & 69,4 & 70,6 & 15 & 74,9 \\
8 & 68,4 & 70,6 & & $72,4 \pm 4,3$ \\
$\begin{array}{c}\text { Moyenne } \\
\pm \text { SEM }\end{array}$ & $73,4 \pm 2,4$ & $69,2 \pm 1,5$ & & \\
\hline
\end{tabular}

(a) Moyenne de deux déterminations

(b) obtenu sur la 8 e semaine de collecte des fèces. 
La méthode de Zilversmit, simple et rapide, connaît un succès mérité. Telle qu'elle a été décrite chez le rat, elle nécessite cependant d'anesthésier l'animal, d'isoler la veine jugulaire ef d'infuser dans l'estomac une solution artificielle simulant un repas, toute opération traumatisante pour l'animal. L'injection dans une veine superficielle, sans anesthésie et l'addition de cholestérol marqué au repas seraient plus satisfaisants sur le plan physiologique. Enfin, cette technique fournit une analyse ponctuelle du CA ne reflétant qu'une période limitée du nycthémère et non une valeur moyenne de celui-ci sur $24 \mathrm{~h}$. Ceci explique probablement la plus grande variabilité des résultats donnés par cette méthode.

La méthode d'équilibre isotopique permet de mesurer l'absorption du cholestérol sur une longue période. Elle permet donc de mieux appréhender le CA d'un animal en équilibre physiologique défini. Contrairement à la méthode du bilan fécal après ingestion d'une dose de cholestérol marqué qui ne prend pas en compte l'excrétion de cholestérol, celui-ci y est mesuré avec précision dans la méthode d'équilibre isotopique ainsi que la sécrétion externe fécale, la transformation en acides biliaires, la sécrétion interne. Cependant, comme pour toute méthode de bilan, la mesure de l'absorption par la méthode d'El n'est valide que si la totalité des molécules radioactives non absorbées est retrouvée dans l'insaponifiable des fèces. Nous avons montré qu'il en est bien ainsi, chez le rat, et qu'il n'existe pas de dégradation bactérienne du noyau stérolique dans de multiples conditions alimentaires (Lutton et Chevallier, 1972). Dans les cas où une telle destruction existe, l'addition d'un indicateur permettant d'en tenir compte est nécessaire. Enfin, cette étude montre que les valeurs du CA du cholestérol fournies par la méthode d'El ne sont pas surestimées. En fait, les différences observées entre nos résultats et ceux publiés Outre-Atlantique (souvent inférieurs de 10 à 20 p. 100) sont dues essentiellement à la nature des régimes utilisés ainsi que nous l'avons montré ailleurs (Mathé et al., 1977). Les valeurs élevées citées parfois (Zilversmit et Hughes, 1974) pour illustrer une possible surestimation de la méthode d'El ont, en fait, été obtenues après addition de taurocholate de sodium dont on sait qu'il stimule fortement l'absorption du cholestérol (Lutton ef Chevallier, 1972).

Commission CNERNA Digestion-Absorption/Association des Physiologistes, Paris 5-6 octobre 1978.

\section{Références}

BORGSTRÖM B., 1968. Quantitative aspects of the intestinal absorption and metabolism of cholesterol and $\beta$-sistosterol in the rat. J. Lipid Res., 9, 473-481.

CHEVALLIER F., MATHÉ D., 1964. Destinée du cholestérol des chylomicrons chez le rat. III. Mouvements de cholestérol $4^{14} \mathrm{C}$ entre les chylomicrons ef la lymphe et le sérum in vivo. Bull. Soc. Chim. biol., 46, 509-527.

COREY J. E., HAYES K. C., 1975. Validation of the dual-isotope plasma ratio technique as a measure of cholesterol absorption in old and new world monkeys. Proc. Soc. exp. Biol. Med., 148, 842-846.

KRITCHEVSKY D., WINTER P. A. D., DAVIDSON L. M., 1974. Cholesterol absorption in primates as determined by the Zilversmit isotope ratio method. Proc. Soc. exp. Biol. Med., 147, 464-466.

LUTTON C., CHEVALLIER F., 1972. Vitesse des processus de renouvellement du cholestérol contenu dans son espace de transfert, chez le rat. III. Modifications et étude cinétique de la méthode d'équilibre isotopique. Biochim. biophys. Acła, 255, 762-779. 
MATHE D., LUTTON C., RAUTUREAU J., COSTE T., GOUFFIER E., SULPICE J. C., CHEVALLIER F., 1977. Effects of dietary fiber and salt mixtures on the cholesterol metabolism of rats. $J$. Nutr., 107, 466-474.

SAMUEL P., CROUSE J. R., AHRENS E. H., 1978. Evaluation of an isotope ratio method for measurement of cholesterol absorption in man. J. Lipid Res., 19, 82-93.

TRABER M. G., OSTWALD R., 1978. Cholesterol absorption and steroid excretion in cholesterol fed guinea pigs. J. Lipid Res., 19, 448-456.

ZILVERSMIT D. B., 1972. A single blood sample dual isotope method for the measurement of cholesterol absorption in rats. Proc. Soc. exp. Biol. Med., 140, 862-865.

ZILVERSMIT D. B., HUGHES L. B., 1974. Validation of a dual isotope plasma radio method for measurement of cholesterol absorption in rats. J. Lipid Res., 15, 565-473. 